\title{
Data Resources and Services at CAsDC
}

\author{
Chenzhou Cui ${ }^{1}$, Boliang $\mathrm{He}^{1}$, Jian Xiao ${ }^{2}$, Ce Yu${ }^{2}$, Jian $\mathbf{L i}^{1}$, \\ Zihuang Cao ${ }^{1}$, Liying $\mathrm{Su}^{3}$, Dongwei Fan ${ }^{1}$, Cuilan Qiao ${ }^{4}$, \\ Changhua Li $^{1}$, Yue Chen ${ }^{1}$, Runtao Wang ${ }^{2}$ and Yongheng Zhao ${ }^{1}$ \\ ${ }^{1}$ National Astronomical Observatories, CAS, Beijing 100012, China \\ email: ccz@bao.ac.cn \\ 2 Tianjin University, Tianjin 300072, China \\ ${ }^{3}$ Beijing University Of Technology, Beijing 100021, China \\ ${ }^{4}$ Central China Normal University, Wuhan 430079, China
}

\begin{abstract}
The Chinese Astronomical Data Center (CAsDC) is a member of World Data System, hosted at National Astronomical Observatories, Chinese Academy of Sciences(NAOC). The CAsDC keeps close collaboration with IVOA, WDS and CODATA. The whole set of LAMOST data, including raw data and data products, are hosted at the CAsDC. Data resources and services of the CAsDC are introduced.
\end{abstract}

Keywords. astronomical data bases: miscellaneous, catalogs, surveys

The Chinese Astronomical Data Center (CAsDC, http://casdc.china-vo.org/) is the scientific data service and infrastructure of NAOC, and has been providing data services to the community since its initiation in the 1980s. In 2012, the CAsDC became a regular member of the World Data System. The Virtual Observatory (VO) is a dataintensive online astronomical research and education environment, taking advantages of advanced information technologies to achieve seamless, global access to astronomical information(Cui \& Zhao 2007). CAsDC is a key service from Chinese Virtual Observatory (China-VO), which is one of the first numbers of the IVOA.

As a cyberinfrastructure for Astronomy research, the CAsDC plays important roles in the following fields:

- Data storage, management and release on behalf of astronomical projects;

- Long-term preservation and access of historical archives;

- Research and development of astronomical data management applications;

- International exchange and sharing of scientific data;

- User support and scientific data based education.

Data resources hosted at the CAsDC can be categorized into two parts, i.e. datasets generated by telescopes and astronomy projects in China, and mirrors of international databases. The Large Sky Area Multi-Object Fiber Spectroscopic Telescope (LAMOST) is a special quasi-meridian reflecting Schmidt telescope for spectroscopic sky surveys. CAsDC is the main entry for LAMOST data products. The whole set of raw observation data and data products, including spectra in FITS format and catalogs are provided. Many IVOA standards and interfaces are supported at the CAsDC, for example, VOTable, SCS, TAP, Aladin, WWT, etc.

This paper is funded by NSFC (U1231108) and MOST of China(2012FY120500).

\section{References}

Cui, C. \& Zhao, Y. 2007, in: W. J.Jin, I. Platais \& M. A.C. Perryman (eds.), IAUS248 Proceedings (Cambridge), p. 563 